\title{
Antibioterapia en Periodoncia. Situación actual I- Antibióticos Sistémicos
}

\author{
FALCÃO COSTA C * \\ MOURA E SÁ A * \\ FARIA ALMEIDA $\mathbf{R} * *$ \\ BASCONES A $* * *$
}

Falcão Costa C, Moura e Sá A, Faria Almeida R, Bascones A. Antibioterapia en Periodoncia. Situación actual. I- antibióticos Sistémicos. Av Periodon Implantol. 2001; 13, 1: 39-47

\begin{abstract}
RESUMEN
En este artículo se hace una revisión bibliográfica sobre la utilización de los antimicrobianos sistémicos en periodoncia. Hoy día existe una gran diversidad de fármacos con capacidad, científicamente fundamentada, de ayudar al periodoncista en su actividad clínica diaria. La evolución de la periodoncia nos permite con la ayuda de tests microbiológicos conocer las bacterias contra las cuales tenemos que actuar, así como los antibióticos más eficaces. Se discute también las dosis actualmente indicadas.
\end{abstract}

\section{PALABRAS CLAVE}

Antibióticos sistémicos, Enfermedad periodontal

\section{INTRODUCCIÓN}

En los últimos años nos hemos encontrado con una evolución significativa en el campo del tratamiento antimicrobíano en periodoncia. En efecto, las nuevas evidencias de la incapacidad del tratamiento mecánico y/o quirúrgico para eliminar toda la etiología bacteriana en algunas formas de enfermedad periodontal así como la necesidad de sustituir una antibioterapia muy empírica (basada en el sentido común y experiencia personal) por una científicamente fundamentada con base en datos de laboratorio, han venido motivando esta constante evolución. Esto va a permitir una selección cada vez mas especifica y mejor orientada del fármaco a utilizar.

Hoy en día disponemos ya de un arsenal considerable de fármacos de administración sistémica o local cuyo objetivo es eliminar las bacterias patógenas para el periodonto.

En esta primera parte de nuestro trabajo vamos a centrarnos solamente en los antibióticos sistémicos, reservando los locales para otro trabajo posterior.

\section{ETIOLOGÍA MULTIFACTORIAL DE LA ENFERIMEDAD PERIODONTAL}

Hay en la literatura un gran número de estudios que demuestran una relación causa-efecto entre la periodontitis y distintos factores. Las conclusiones obtenidas nos dicen que la etiología es multifactorial. Factores tan inespecíficos como la raza (principalmente en personas de origen filipino o de raza negra), el sexo (mas frecuente en los hombres) o la edad avanzada se añaden a otros mas específicos como son el tabaco o la diabetes, constituyendo importantes marcadores de mayor riesgo de enfermedad periodontal más severa.

La misma relación esta ya documentada con la presencia en la placa subgingival de bacterias como P.gingivalis, P.intermedia o F.nucleatum.

Otras situaciones como son: un status socioeconómico o educacional bajo, el vivir en zonas rurales $\circ$ tener una deficiente alimentación (falta de vit. A, B, C, D, calcio o proteínas) están siendo citadas también

\footnotetext{
* Alumno de la Facultad de Odontologia de la Universidad de Oporto

** Master de Periodoncia. Facultad de Odontologia de la Universidad Complutense de Madrid

*** Catedrático de Medicina Bucal y Periodoncia. Universidad Complutense de Madrid
} 
como factores etiológicos relevantes. Nada sorprendente es la gran documentación que relaciona la gravedad y frecuencia de la enfermedad con la mala higiene bucal, tantas veces también asociada a la ausencia de cuidados dentales profesionales.

Mas recientemente, han surgido estudios que demuestran la existencia de una susceptibilidad genética en algunos pacientes que va a condicionar un mayor riesgo de que sufran de periodontitis (de inicio precoz). Es sobre este campo de la Genética que se investiga cada vez más y es ahí donde están probablemente los mayores desafíos para la Periodoncia y toda la Medicina en general $(1,2,3)$.

Como conclusión, hoy en día podemos dividir los factores etiológicos más importantes en la enfermedad periodontal en tres grandes grupos (esquema 1) $(1,2,3)$.

\section{MICROBIOLOGÍA DE LA ENFERIMEDAD PERIODONTAL}

La búsqueda de agentes etiológicos para la enfermedad periodontal no es reciente, pues solo en los años 60 se estabilizó una relación causa-efecto entre la presencia de placa bacteriana y la presencia de enfermedad $(4,5)$.
No se valoraba la variabilidad de la composición de la placa entre distintos individuos y dentro del mismo individuo en diferentes lugares. Diferentes estudios hechos en los años 70 han concluido de una forma general que las localizaciones con enfermedad periodontal activa tenían una microbiota especifica y diferente de las localizaciones sanas en el mismo individuo.

Después de años de investigación tenemos hoy referenciadas algunas bacterias que presentan una gran relación con la aparición de la periodontitis, pudiendo considerarse como agentes etiológicos. Son ejemplos el A.actinomycetemcomitans y la $P$. gingivalis (tabla 1), así como las espiroquetas, estas ultimas como las principales patógenas en la PUNA. Otras como el F.nucleatum, P.intermedia, B.forsythus, E.corrodens, P.micros, entre otras, necesitan de una mejor definición de su importancia en la patogénesis de la enfermedad, aunque su presencia en varias formas de periodontitis este confirmada. (4)

La demostración de la capacidad que tienen algunas bacterias (A.a. y P.gingivalis) de invadir las células epiteliales asociada a las dificultades del punto de vista anatómico del tratamiento convencional han venido a confirmar los resultados de algunos estudios que demuestran la ineficacia del raspado y alisado radicular o incluso del tratamiento quirúrgico en eliminar estas bacterias. $(4,5)$

\begin{tabular}{|c|c|c|c|}
\hline & $\begin{array}{l}\text { Características } \\
\text { generales }\end{array}$ & $\begin{array}{l}\text { Principales factores de } \\
\text { virulencia }\end{array}$ & $\begin{array}{l}\text { Principales enfermedades } \\
\text { asociadas }\end{array}$ \\
\hline $\begin{array}{l}\text { Actinobacilus } \\
\text { Actinomycetemcomitans }\end{array}$ & $\begin{array}{l}\text { - bacilo Gram-negativo } \\
\text { - inmóvil } \\
\text { - capnofilico }\end{array}$ & $\begin{array}{l}\text { - leucotoxina/endotoxina } \\
\text { - colagenasa } \\
\text { - degradación de lg's } \\
\text { - inducción de reabsorción } \\
\text { del hueso } \\
\text { - capacidad de invasión } \\
\text { de cels. epiteliales. } \\
\text { - capacidad de inhibir los } \\
\text { fibroblastos }\end{array}$ & $\begin{array}{l}\text { - periodontitis juvenil } \\
\text { localizada } \\
\text { - periodontitis } \\
\text { prepuberal } \\
\text { - algunas formas de perio- } \\
\text { dontitis del adulto }\end{array}$ \\
\hline $\begin{array}{l}\text { Porphyromonas } \\
\text { Gingivalis }\end{array}$ & $\begin{array}{l}\text { - bacilo Gram-negativo } \\
\text { - anaerobio estricto } \\
\text { - inmóvil } \\
\text { - } \text { asacarolítico }\end{array}$ & $\begin{array}{l}\text { - colagenasa } \\
\text { - endotoxina } \\
\text { - fibrinolisina } \\
\text { - fosfolipasa a2 } \\
\text { - capacidad de inhibir los } \\
\text { fibroblastos } \\
\text { - inducción de reabsorción } \\
\text { del hueso } \\
\text { - degradación de lg's } \\
\text { - capacidad de invasión } \\
\text { de cels. epiteliales }\end{array}$ & $\begin{array}{l}\text { varias formas de perio- } \\
\text { dontitis del adulto } \\
\text { - periodontitis de inicio } \\
\text { precoz }\end{array}$ \\
\hline
\end{tabular}

Tabla 1. Características de los principales periodontopatógenos. 
Esto ha llevado a un intento de utilizar fármacos que alcanzasen concentracion mínima inhibitoria a nivel del fluido crevicular, fondo de bolsas, tejido conectivo subgingival y otras zonas de la cavidad bucal (mucosa masticatoria, por ejemplo). (5)

\section{ANTIBIÓTICOS SISTÉMICOS VS ANTIBIÓTICOS LOCALES}

La facilidad de acceder al lugar de la infección en el tratamiento de la enfermedad periodontal nos permite escoger como forma de administrar antibióticos no solo la vía sistémica sino también la aplicación local. Cada uno de los métodos tiene ventajas y desventajas específicas.

La utilización de la vía sistémica posibilita tratar múltiples bolsas simultáneamente y también alcanzar otros lugares de la cavidad bucal(dorso de lengua, mucosa bucal, pilares amigdalinos, etc) que pueden funcionar como reservorios de bactérias. $(6,7,8)$. Esta característica favorable tiene también desventajas como reacciones adversas más frecuentes (peligro de no utilización por parte del paciente) y un mayor riesgo de causar resistencias bacterianas, lo que se traduce por limitaciones a nivel de dosis y terapéutica a utilizar. $(6,7,8)$.

Los antibióticos administrados localmente permiten a su vez, alcanzar concentraciones dentro de la bolsa periodontal 10-100 veces superiores que las conseguidas con la vía sistémica y además con mucho menor riesgo de provocar reacciones adversas o resistencias bacterianas en otros lugares del organismo. Siendo así tenemos la posibilidad de usar fármacos de amplio espectro, cuya utilización sistémica no seria muy recomendable. $(7,9)$.

Sin embargo, y cuando se comparó con la vía sistémica, las técnicas más eficaces de aplicación local de antibióticos dan más trabajo, son mas lentas y debido a su restringido campo de acción (bolsa) aumentan el riesgo de recidiva (9).

Analizando cuidadosamente los pros y los contras del uso de antibióticos en los tratamientos periodontales, la conclusión que se puede sacar es que solo se deben utilizar en situaciones de clara necesidad, independientemente de la vía escogida.

\section{TETRACICLINAS}

Constituyen un grupo de agentes de amplio espectro eficaces contra muchas especies Gram-negativas, incluyendo también bactérias periodontopatógenas como A.actinomycetemcomitans. Actúan por inhibición de la síntesis proteica, siendo entonces primariamente bacteriostáticas $(6,10,11,12)$. La doxiciclina y la minociclina son, hoy en día, las más usadas en periodoncia.

Las tetraciclinas tienen un alto porcentaje de absorción cuando se administran por vía oral, gran unión a las proteínas plasmáticas y elevada liposolubilidad. $\mathrm{Su}$ semivida permite administraciones $1-2$ veces al día y las concentraciones alcanzadas en el fluido gingival son 2-4 veces superiores a las séricas (10).

Provocan con alguna frecuencia reacciones adversas como intolerancia gastrointestinal, diarreas, náuseas $(6,8,10,11)$ y pueden inducir la aparición de resistencias bacterianas y sobreinfecciones por Candida o enterobacterias durante el tratamiento $(6,10,12)$. Se encuentran descritas resistencias a la tetraciclina para varios géneros de anaerobios como Prevotella, Fusobacterium, Porphyromonas, y en algunos pacientes para A.a., debido al consumo regular del antibiótico (13). No debe ser administrada en pacientes embarazadas, ni conjuntamente con leche, sus derivados y/o antiácidos $(10,11)$.

Las tetraciclinas tienen, además de la actividad antibacteriana, otras propiedades que aumentan los beneficios de su utilización: acción antiinflamatória, inhibición de la colagenasa y de la reabsorción del hueso, capacidad de promover la unión de los fibroblastos a la superficie del diente y capacidad de unirse a los dientes siendo liberadas lentamente durante algún tiempo $(6,10,12)$.

En un estudio en pacientes con periodontitis crónica del adulto, Lindhe y cols. (14) utilizando tetraciclina (dosis de $250 \mathrm{mg} / 4$ veces al día/ 14 días $+250 \mathrm{mg} /$ día durante el resto del estudio-12 meses) juntamente con raspado y alisado radicular obtuvieron, en comparación con un tratamiento control de $\mathrm{R}$ y A + placebo, mejores resultados al nivel de disminución de las profundidades de sondaje, índice gingival, ganancias de inserción y reducción del número de microorganismos móviles. Estos resultados son contrarios a los obtenidos en otros estudios $(15,16)$ con el mismo diseño y también en pacientes con periodontitis crónica, en los cuales no hubo diferencias significativas entre los pacientes tratados con raspado y alisado solo o con la administración coadyuvante de tetraciclina.

Los efectos de las tetraciclinas cuando se administran por vía sistémica fueron también estudiados en pacientes con periodontitis juvenil localizada, habiéndose verificado en varios de los estudios revisados una disminución de los niveles de A.a. y mejo- 
ras clínicas $(17,18,19)$, después de tratamientos combinados con R y A o con.cirugía periodontal. Sin embargo, hay referencias en la literatura de recidivas de la enfermedad periodontal y de los niveles de A.a. después de tratamientos con tetraciclina (20).

Siendo un antibiótico de amplio espectro su actividad fue también estudiada en pacientes con periodontitis refractaria. En estos estudios y utilizando tetraciclina (250mg/4 veces al día/14-21 días) o doxiciclina (100 $\mathrm{mg} /$ día/2l días) como terapia complementaria al $\mathrm{R}$ y A se verificó concordancia de resultados en la reducción de profundidad de sondaje y disminución del numero de localizaciones con enfermedad activa $(21,22,23)$. Bacteriológicamente, si hay estudios donde se pudo comprobar la eficacia de las tetraciclinas sobre A.a. $(22,24)$ o sobre P.gingivalis y P.intermedia $(24,25)$, hay otros donde no hubo alteraciones en los niveles de A.a. (25) o de P.g. y P.i. (22) después del tratamiento sistémico con este fármaco.

\section{METRONIDAZOL}

Fármaco bactericida (del grupo de los nitroimidazoles) que actúa al nivel de la síntesis de DNA, a través del grupo nitro reducido por ferredoxinas y por otros compuestos bacterianos (11). Presenta en consecuencia una toxicidad selectiva con relación a bactérias anaerobias estrictas (P.gingivalis, P.intermedia, F.nucleatum, Bacteroides) y protozoos, pero no es activo contra organismos aeróbicos o facultativos (A.actinomycetemcomitans, E.corrodens) $(6,10,11,13)$. Hay sin embargo estudios que hablan de la existencia de algunos bacilos Gram-negativos anaerobios (especialmente de P.melanogénica y cepas de P.micros) resistentes a los nitroimidazoles $(10,13)$.

Administrado normalmente en 3 tomas diarias (cada 6-8 horas) presenta generalmente buena tolerancia, pudiendo surgir náuseas, vómitos 0 diarreas $(6,10,11,13,26)$. No induce la aparición de resistencias bacterianas durante el tratamiento, debiendo evitarse su uso en pacientes embarazadas $(10,11)$.

Lindhe y col. (27) demostraran que el metronidazol administrado por vía sistémica conseguía diminuir o eliminar los signos clínicos de enfermedad periodontal, siendo activo sobre espiroquetas y bastones móviles (conclusiones confirmadas por estudios posteriores de otros autores) $(28,29,30)$. Verificaron también que la disminución de los niveles de espiroquetas se mantenía mucho tiempo después de la permanencia del fármaco en el organismo. Sin embargo, y después del tratamiento con metronidazol, hay algunas bactérias (P.gingivalis, P.intermedia, Espiroquetas, F.nuclea- tum) con tendencia a la recidiva, lo que probablemente se debe a una incompleta limpieza o rápida recolonización de los lugares tratados $(29,31,32)$.

Es también curioso verificar que a pesar de la toxicidad selectiva sobre anaerobios, una terapéutica con metronidazol sistémico en dosis de 200mg/día/ lOdías (33) obtuvo disminución de A.a. en pacientes con Periodontitis juvenil localizada (probablemente debido al metabolito hidroxil)(33,34). Clínicamente hubo disminución de profundidades de sondaje y del índice gingival. En este mismo estudio, los resultados obtenidos con la tetraciclina fueron peores que con el metronidazol.

La administración de metronidazol puede también reducir él numero de dientes con necesidad de cirugía periodontal (29).

Parece ser conclusión generalizada que este fármaco es más eficaz en pacientes que tengan altas proporciones de espiroquetas. Algunos estudios $(29,29)$ sugieren que la mejor manera de usar el metronidazol es en tratamiento combinado con raspado y alisado radicular.

En casos de periodontitis rápidamente progresiva, el tratamiento complementado con este antibiótico parece mejorar la cicatrización por un periodo hasta 6 meses, cuando se comparó con el raspado y alisado solo (26).

\section{AMOXICILINA + AC.CLAVULANICO}

Tiene un amplio espectro de acción que incluye la gran mayoría de las bacterias anaerobias estrictas, incluyendo también las productoras de $\beta$-lactamasas $(10,13)$. Sus características farmacológicas permiten la administración cada 8-12 horas y una buena penetración en los tejidos y fluidos gingivales (10). Tiene una acción bactericida y normalmente no provoca reacciones adversas, aunque ocasionalmente puedan ocurrir diarreas $(8,10)$.

En un estudio con pacientes con Periodontitis refractaria, Magnusson y cols. (35) utilizando dosis de 250mg/3 veces al día/14 días de amoxicilina+ác. clavulanico combinado con raspado y alisado radicular, obtuvieran disminución de la pérdida de inserción, de la profundidad de sondaje y del número de lugares con enfermedad activa a los 9 meses. Usando el mismo protocolo, pero a 24 meses, los mismos autores han verificado ganancia de inserción en algunos lugares. Sin embargo, a partir de los 12-15 meses empezaran a observar un nuevo aumento de las pro- 
fundidades de sondaje y tendencia de una nueva pérdida de inserción (36).

Otros estudios $(37,38,39)$ en pacientes con periodontitis refractaria y utilizando el mismo tipo de terapéutica, obtuvieron resultados semejantes en términos de reducción de las profundidades de sondaje, mayor número de lugares con ganancia de inserción y disminución de lugares con enfermedad activa.

Collins y cols. (40) verificaron la eliminación de P.gingivalis en casi todos los pacientes que estaban infectados antes del tratamiento, utilizando un tratamiento combinado de amoxicilina+ác. clavulanico y raspado y alisado radicular. La mejoría clínica obtenida se ha mantenido en una revaluación 3 años después.

\section{CLINDAMIICINA}

Es un macrólido del tipo de la lincosasamina, de acción bacteriostática, que actúa sobre la subunidad 50 s de los ribosomas, alterando la síntesis proteica $(10,41)$. Su espectro de acción incluí la $P$. Gingivalis, $P$. Intermedia y F. Nucleatum, siendo inactivo sobre la A.actinomycetemcomitans $(10,13)$. Su semivida permite administraciones entre cada 6-8 horas, teniendo concentraciones en el fluido crevicular semejantes a las séricas $(8,10,41)$. Entre sus efectos secundarios se destacan los de tipo digestivo (que incluyen náuseas, vómitos y diarreas) y el principal que es la posibilidad de presentación de colitis pseudomembranosa, que puede ocurrir durante el tratamiento, normalmente entre el cuarto y el noveno día, o algunas semanas después de finalizar la administración del fármaco. Su eliminación se hace principalmente por vía renal $(6,10,13,41)$.

En un estudio realizado por Gordon y cols. (42) en 13 pacientes que presentaban una periodontitis refractaria, se intentó determinar cual es el efecto de la administración de clindamicina como complemento al raspado y alisado radicular para el tratamiento de esta forma de enfermedad periodontal. La posología adoptada ha sido de $150 \mathrm{mg} 4$ veces/día durante 7 días. Los autores han verificado que la clindamicina aumentaba la eficacia del tratamiento mecánico por la disminución del número de lugares activos de enfermedad (pasaron de un porcentaje de 10,7\% al $0,5 \%$ ) y por la mejoría de parámetros clínicos como inflamación gingival, hemorragia, eritema, supuración y profundidad de bolsa a los 12 meses post-tratamiento. La flora cultivada antes del tratamiento con clindamicina consistía esencialmente de Gram-negativos (P.intermedia y P. Gingivalis, principalmente), y la cantidad de las especies cultivadas se mantuvo prác- ticamente igual a los 12 meses post-tratamiento cuando se comparó con el periodo pre-tratamiento (43).

\section{TERAPIA COMBINADA}

El uso concomitante de fármacos es muchas veces esencial para obtener el objetivo terapéutico pretendido (44). La asociación de antimicrobianos tiene como principal ventaja el aumento del espectro de acción que el que se consigue por cada fármaco individualmente $(6,8)$.

También nos permite prevenir la aparición de resistencias bacterianas y disminuir la dosis de cada fármaco a administrar, pues hay un sinergismo cuando se asocian distintos antibióticos $(6,8)$.

Por otra parte, aumentamos también el riesgo de aparición de reacciones adversas y el peligro de tener interacciones indeseables entre substancias que sean antagonistas. Como ejemplo, tenemos el metronidazol que siendo bactericida no debe usarse con tetraciclina (bacteriostástico), pues el primero actúa sobre la división celular, que es impedida por esta última (6).

En un estudio de Pavicic y col. (45) se intentó evaluar los efectos clínicos y microbiológicos resultantes de la utilización de tratamiento mecánico y tratamiento antibiótico (250 mg de metronidazol + $375 \mathrm{mg}$ de amoxicilina $3 \mathrm{x}$ dia/ 7 días) en 48 pacientes con periodontitis, evaluando los resultados del A.a. a los 3 y 24 meses después del tratamiento. Los autores concluyeron que la combinación de los dos tipos de tratamiento fue eficaz en la eliminación del A.actinomycetemcomitans y P.gingivalis durante un largo período de tiempo y que la recolonización se presentó raramente. Sin embargo, verificaron que la eliminación del A.a. fue acompañada de una mejoría del estado periodontal de los pacientes, incluso a los 24 meses después del tratamiento. Por otra parte, el estudio también ha demostrado la ineficacia de este tratamiento cuanto a la supresión del $P$. intermedia. Las mismas conclusiones han sido verificadas por Van Winkelhoff y cols. (46), utilizando el mismo tipo de posología para el metronidazol y amoxicilina.

Kornman y cols. (47) no han obtenido diferencias clínicas y microbiológicas entre un tratamiento con metronidazol +amoxicilina y el tratamiento con metronidazol + amoxicilina/ác.clavulanico.

La asociación metronidazol + ciprofloxacina fue estudiada en 17 pacientes que presentaban periodontitis refractaria (48), señalándose mejorías clínicas y una disminución del número de los periodonpatógenos. 


\section{CONCLUSIONES}

Hoy día es unánime que la enfermedad periodontal tiene una etiología multifactorial. En efecto, existe un componente genético que hace el $10 \%$ de la población resistente y otro $10 \%$ muy susceptible. Además del componente genético hay también un factor imunológico que va influir en la enfermedad periodontal, indiscriminada, aleatoria y sin ningún tipo de base científica lo que ha motivado la aparición de resistencias bacterianas. Hoy se intenta utilizarlos correctamente, solo cuando sean estrictamente necesarios y dirigidos para las bacterias que efectivamente producen la enfermedad en un determinado individuo. La forma conseguirlo es mediante el uso imprescindible de tests microbiológicos que se deben hacer

\begin{tabular}{|c|c|c|}
\hline Tipo de enfermedad & Antibiótico & Posología \\
\hline $\begin{array}{l}\text { Periodontitis juvenil } \\
\text { localizada }\end{array}$ & $\begin{array}{l}\text { Metronizadol o } \\
\text { Metronizadol + Amoxilina }\end{array}$ & $\begin{array}{l}250 \mathrm{mg} \text { cada } 8 \text { horas } / 7 \text { días } \\
250 \mathrm{mg}+375 \mathrm{mg} \text { cada } 8 \text { horas } / 7 \text { días }\end{array}$ \\
\hline $\begin{array}{l}\text { Periodontitis refractaria del } \\
\text { adulto }\end{array}$ & $\begin{array}{l}\text { Metronizadol o } \\
\text { Amoxilina + Ác. clavulanico o } \\
\text { Metronizadol + Amoxilina }\end{array}$ & $\begin{array}{l}250 \mathrm{mg} \text { cada } 8 \text { horas } / 7 \text { días } \\
250 \mathrm{mg} \text { cada } 8 \text { horas / } 14 \text { días } \\
250 \mathrm{mg}+375 \mathrm{mg} \text { cada } 8 \text { horas } / 7 \text { días }\end{array}$ \\
\hline
\end{tabular}

\section{Tabla 2.}

teniendo como resultado, que individuos con una inmunidad diminuida sean más susceptibles a sufrir la enfermedad y a presentar un avance más rápido.

Sin embargo, esta claro que para que exista la enfermedad tiene que haber bacterias. Sin ellas no va a ocurrir periodontitis, aunque el individuo sea genéticamente y imunológicamente comprometido. Se sabe también que las bacterias no pueden ser totalmente eliminadas con el tratamiento convencional de $\mathrm{R}$ y $\mathrm{A}$ radicular y/o con cirugía. Por eso, el uso de antibióticos está siendo cada vez más propuesto como complementario en los tratamientos periodontales. En un principio eran utilizados de una forma siempre que pretendamos utilizar el auxilio de la antibioterapia.

Hoy existe un conjunto de fármacos que, después de años de estudios, esta comprobado que son eficaces en el combate de los periodontopatógenos y en consecuencia de la enfermedad. Parece ser incluso posible asociar la presencia de determinadas bacterias a distintos tipos de periodontitis, permitiendo así, utilizar en cada situación de enfermedad periodontal el antibiótico más eficaz (tabla 2).

Actualmente, parece que son necesarios nuevas investigaciones para conocer la respuesta a antibióti-

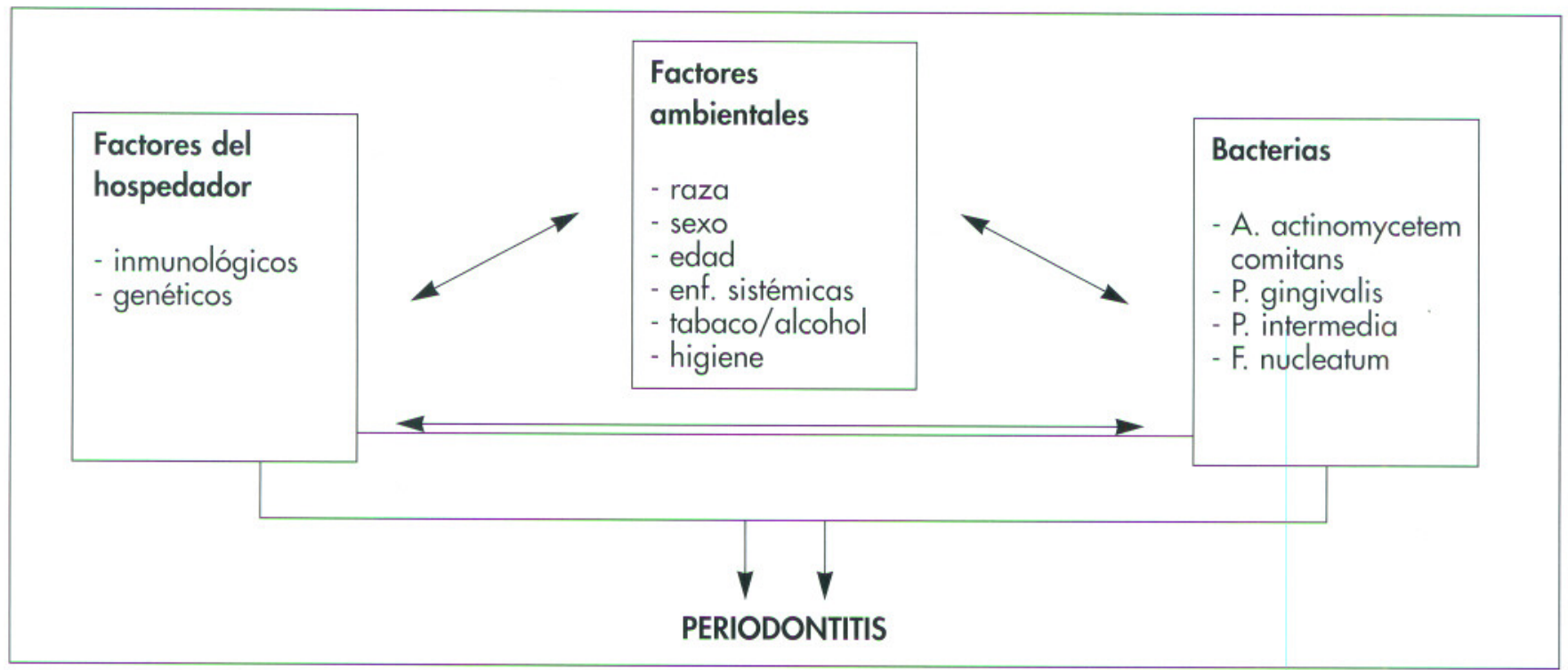

Esquema 1. Representación de la etiología multifactorial de la enfermedad periodontal 
cos poco estudiados como la Azitromicina y también a otros tipos de asociaciones de fármacos en el tratamiento de la enfermedad periodontal.

\section{SUMMMARY}

This review article focuses on the use of systemic antibiotics in the management of periodontal diseases. Nowadays, we have a large variety of drugs available to help us in our daily practice. The constant evolution and never ending scientific experiments on this subject provide not only the possibility to identify the bacterial flora involved in periodontal diseases, but also to choose the best antibiotic for each situation.

\section{KEY WORDS}

Systemic antibiotics, periodontal disease

\section{CORRESPONDENCIA:}

A Bascones Martinez

Boix y Morer, $6,1^{\circ}$

28003 Madrid

\section{BIBLIOGRAFÍA}

1. Papanou PN, Lindhe J. Epidemiology of periodontal disease; Clinical Periodontolgy and Implant Denstistry; 69-101 Munksgaard 1995.

2. Bascones A. Epidemiologia de la enfermedad periodontal; Periodoncia: Diagnostico y tratamiento de la enfermedad periodontal; 131-132.

3. Carranza F, Newman. Periodontologia Clinica. Epidemiologia de los transtornos gingival y periodontal; pág 79-83.

4. Socransky SS, Haffajee AD. Microbilogy of periodontal disease; Clinical Periodontolgy and Implant Denstistry; 138-200 Munksgaard 1995.

5. Haffajee $A D$, Socransky SS. Microbial etiological agents of destructive periodontal disease. Periodontol 2000 1994; 5: 78-111.
6. Mombelli A. Antibiotics in periodontal therapy; Clinical Periodontology and Implant Dentistry; 488-510 Munksgaard 1995.

7. Greenstein G, Polson A. The role of local drug delivery in the management of periodontal diseases: a comprehensive review. J Periodontol 1998; 69: 507-520.

8. Van Winkelhoff AJ, Rams TE, Slots J. Systemic antibiotic therapy in periodontics. Periodontol 2000 1996; 10: 4578.

9. Villarverde RG, Blanco CJ, Ramos BI, Batalla VP. Aplicación subgingival de antimicrobianos en periodoncia. Avances en Periodoncia e Implantologia oral 1999; 11: 103-116.

10. García Sanchez JE, Fresnadillo MMJ, García Sanchez E. Microbiologia bucal y antimi crobianos. Ediciones Avances.

11. Bascones A, Arce BL. Antibioticos y Antimicrobianos en Periodoncia; Tratado de Odontologia: Ed. Avances. Madrid,2000.

12. Seymour RA, Hensman PA. Tetracyclines in the management of periodontal diseases. A review. J Clin Periodontol 1995; 22: 22-35.

13. Dubreuil L, Bascones A. Infecciones periodontales: papel de los anaerobios estrictos, importancia de las Blactamasas, elección de un antibiótico. Avances en Periodoncia 1994; 6: 99-108.

14. Lindhe J, Lijlenberg B, Adielson B. Effect of tetracycline therapy on human periodontal disease. J Clin Periodontol 1983; 10: 590-601.

15. Chaves E, Ryerson C, Snyder B, Jeffcoat M. Effect of tetracycline therapy on specific bacterial antigens and bone height. J Dent Res 1995; 26:abstr 114.

16. Schroeder KL, Ramamurthy NS, Sczepanek KA. Lowdose doxycycline prevents attachment loss in adult periodontitis. J Dent Res 1992; 71: 758, abstr 1936.

17. Christersson LA, van Winkelhoff AJ, Zambon JJ, de Graaff J, Genco RJ. Systemic antibiotic conbination therapy in recalcitrant and recurrent localized juvenile periodontitis. J Dent Res 1989; 68:197, abstr128.

18. Mandell RL, Socransky SS. Microbiological and clinical effects of surgery plus doxycycline on juvenile periodontitis. J Periodontol 1988; 59:373-379.

19. Slots J, Rosling BG. Supression of the periodontophatic microflora in localized juvenile peirodontitis by systemic tetracycline. J Clin Periodontol 1983; 10: 465-486. 
20. Lindhe J. Treatment of localized juvenile periodontitis. In: Genco RJ, Mergenhagen SE, ed. Host-parasite interactions in periodontal diseases. Washington, DC: Am Soc Microbiol, 1981: 382-394.

21. Fiehn N-E, Westergaard J. Recurrent periodontitis: effect of doxycycline on the subgingival microflora. J Dent Res 1989; 68:916, abstr 400 .

22. Haffajee AD, Dzink JL, Socranky SS. Effect of modified Widman flap surgery and systemic tetracycline on the subgingival microbiota of periodontal lesions. J Clin Periodontol 1988; 15: 255-262.

23. Rams TE, Keyes PH, Wright WE, Howard SA. Long-term effects of microbiologically modulated periodontal therapy on advanced adult periodontitis. J Am Dent Assoc 1985; 111: 429-441.

24. Bragd L, Wikstrom M, Slots J. Clinical and microbiological study of "refractory" adult periodontitis. J Dent Res 1985;64: 234, abstr 538 .

25. Kulkarni GV, Lee WK, Aitken S, Birek P, McCulloch CAG. A randomised placebo-controlled trial of doxycycline: effects on the microflora of recurrent periodontitis lesions in high risk patients. J Periodontol 1991;62: 197202.

26. Soder PO, Frithiof L, Wikner S, Wouters FR, Nedlich V, Soder B, Rubin B. The effects of metronidazole in tretment of young adults with severe periodontitis. J Dent Res 1989; 68: 710, abstr 86 .

27. Lindhe J, Lidhenberg B. Use of metonidazole as a probe in the study of human periodontal disease. J Clin Periodontol 1983; 10: 100-112.

28. Noyan U, Yilmaz S, Kuru B, Kadir T, Acar O, Buget E. A clinical and microbiological evaluation of systemic and local metronidazole delivery in adult periodontitis patients. J Clin Periodontol 1997; 24: 158-165 Munksgaard 1997.

29. Loesche WJ, Giordano JR, Hujoel P, Scharcz J, Smith BA. Metronidazole in periodontitis. III. Reduced need for surgery. J Clin Periodontol 1992; 19: 103-112.

30. Soder PO, Frithiof L. The effect of systemic metronidazole after non-surgical treatment in moderate an advanced periodontitis in young adults. J Periodontol 1990; 61: 281-288.

31. Loesche WJ, Giordano JR. Metronidazole in periodontitis. V. Debridement Should precede medication. Compendium 1994; 15: 1198-1218.
32. Loesche WJ, Syed SA, Morrison EC, Kerry GA, Higgins T, Stoll J. Metronidazole in periodontitis. I. Clinical and bacteriological results after 15 to 30 weeks. J Periodontol 1984; 55: 325-335.

33. Saaxen L, Asikainen S. Metronidazole in the treatment of localized juvenile periodontitis. J Clin Periodontol 1993; 20: 166-171.

34. Jousimies-Somer H, Asikainen S, Suomala P, Summanen P. Activity of metronidazole and its hydroxymetabolite. Oral Microbiol Immunol 1988; 3: 32-34.

35. Magnusson I, Clark WB, Low SB, Marunaik J, Marks RG, Walker CB. Effect of non-surgical periodontol therapy combined with adjunctive antibiotics in subjects with "refractory" periodontal disease. I. Clinical results. J Clin Periodontol 1989; 16: 647-653.

36. Magnusson I, Low SB, McArthur WP, Marks RJ, Walker CB, Maroniak J, Taylor M, Padget P, Jung J, Clark WB. Treatment of subjects with refractory periodontal disease. J Clin Periodontol 1994; 21: 628-637.

37. Haffajee AD, Dibart S, Kent RL jr, Socransky SS. Clinical and microbiological changes associated with the use of 4 adjunctive systemically administered agents in the treatment of periodontal infections. J Clin Periodontol 1995; $22:$ 618-627.

38. Low SB, Magnusson I, Clark WB, Walker CB, Marks RG. Changes in attachment level in subjects with progressive "refractory" periodontitis. J Dent Res 1988; 67: 157, abstr 357.

39. Newman MG, Kornman KS, Flemming TF, Nachnani S, Alvarado R. Tretment of refractory periodontitis with Augmentin. J Dent Res 1989; 68: 917, abstr 404.

40. Collins JG, Offenbacher S, Arnold RR. Effects of a combination therapy to eliminate Porphyromonas Gingivalis in refractory periodontitis. J Periodontol 1993; 64: 998-1007.

41. Calatayud J, Sicilia AM, Lucas M. Revision de la clindamicina. Avc Odontoestomatologia 1988; 4: 397-402.

42. Gordon J, Walker CB, Hovliaras C, Socransky SS. Efficacy of clindamycin hydrochloride in refractory periodontitis: 24 month results. J Periodontol 1990; 61: 686- 691.

43. Walker C, Gordon J. The effect of clindamycin on the microbiota associated with refractory periodontitis. J Periodontol 1990; 61: 692-698.

44. Goodman A, Gilman. The farmacological basis of therapeutics. Macmillan Publish company, 1995. 
45. Pavicic MP, van Winkelhoff AJ. Microbiological and clinical effects of metronidazole and amoxicillin in Actinobacillus actinomycetencomitans-associated periodontitis: a 2 year evaluation. J Clin Periodontol 1994; 27: 107-112.

46. van Winkelhoff $\mathrm{AJ}$, Tijhof CJ, de Graaff J. Microbiological and clinical results of metronidazole plus amoxicillin therapy in Actinobacillus actinomycetencomitans associated periodontitis. J Periodontol 1992; 63: 52-57.

47. Kornman KS, Newman MG, Flemming T, Alvarado R, Nachnani S. Treatment of refractory periodontitis with metronidazole plus amoxicillin or Augmentin. J Dent Res 1989; 68: 917, abstr 403.

48. Rams TE, Feik D, Slots J. Ciprofloxacin/Metronidazole treatment of recurrent adult periodontitis. J Dent Res 1992; 71:319, abstr 1708 .

49. Gordon JM, Walker CB, Lamster I, West T, Socransky SS, Seiger M, Fasciano R. Efficacy of clindamycin hydrocloride in refractory periodontitis - 12 months results. J Periodontol 1985; 56 (suppl): 75-80.
50. Kornman KS, Robertson PB. Clinical and microbiological evaluation of therapy for juvenile periodontitis. J Periodontol 1985; 56: 443-446.

51. Listgarten MA, Lindhe J, Helldén L. Effect of tetracycline and/or scaling on human periodontal disease. Clinical, microbiological, and histopathological observations. J Clin Periodontol 1978; 5: 246-271.

52. van Winkelhoff AJ, Rodenburg JP, Goene RJ, Abbas F, Winkel EG, de Graaff J. Metronidazole plus amoxicillin in the treatment of Actinobacillus actinomycetencomitans associated periodontitis. J Clin Periodontol 1989; 16: 128-131.

53. Loesche WJ, Grossman N, Giordano J. Metronidazole in periodontitis. IV. The effeect of patient compliance on treatment parameters. J Clin Periodontol 1993; 20: 96104.

54. Walker CB. The acquisition of antibiotic resistance in periodontal microflora. Periodontol 2000 1996; 10: 179188. 